Wien klin Mag 2016 · 19:1-2 DOI 10.1007/s00740-016-0095-4

Online publiziert: 28. Januar 2016

๑) Springer-Verlag Wien 2016
Alois Sillaber

Springer, Wien, Österreich

\section{Open Access publizieren im "Wiener klinischen Magazin“}

\author{
Wissenschaftler in Österreich können ab \\ 2016 in über 1600 Springer-Zeitschriften - \\ darunter auch dem „Wiener klinischen \\ Magazin" - Open Access publizieren
}

Springer und das Bibliothekskonsortium in Österreich (Kooperation E-Medien Österreich/KEMÖ) haben im Herbst 2015 ein weitreichendes, neues Lizenzmodell „Springer Compact“ mit dreijähriger Laufzeit ab Januar 2016 vereinbart. Springer Compact verbindet die Nutzung von Inhalten auf SpringerLink mit der Möglichkeit, Open Access (OA) zu publizieren. Die Konsortialmitglieder erhalten Zugriff auf über 2000 SpringerZeitschriften und können in über 1600 Springer Hybrid-Zeitschriften (wie z. B. dem „Wiener klinischen Magazin“) Open Access publizieren.

Springer hat ein vergleichbares Modell mit den niederländischen Universitäten, der Max Planck Gesellschaft und in Großbritannien vereinbart. Das neue Modell bietet Wissenschaftlern und Wissenschaftlerinnen hervorragende Möglichkeiten, ihre Forschungsergebnisse in einem qualitativ hochwertigen und breiten Zeitschriftenportfolio Open Access zu publizieren. Sie können sich auf das Publizieren konzentrieren und müssen sich nicht um die finanziellen Rahmenbedingungen und administrativen Anforderungen im Hinblick auf OA-Mandate kümmern.

Gültig ist diese Regelung für alle Manuskripte, die nach dem 1.1.2016 akzeptiert werden, d. h. es können auch Artikel, die schon vor 2016 eingereicht wurden, berücksichtigt werden.

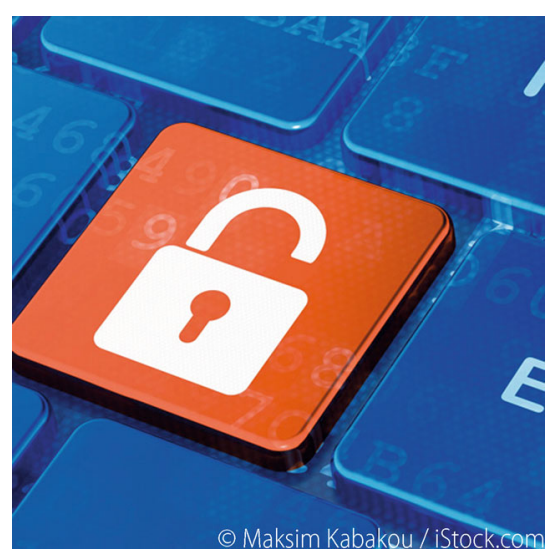

Bei Fragen $\mathrm{zu}$ den Möglichkeiten der OA-Publikation im „Wiener klinischen Magazin“ wenden Sie sich bitte an: oa.verification@springer.com. Weiterführende Informationen finden Sie unter: www.springer.com/de/openaccess/springer-open-choice/agreements/ 750588

Alois Sillaber

Springer Wien, Österreich

\section{Korrespondenzadresse}

\section{Dr. A. Sillaber}

Springer

Wien, Österreich

alois.sillaber@springer.at 
Hier steht eine Anzeige.

Springer 